\title{
Dual Front Evolution Model and Its Application in Medical Imaging
}

\author{
Hua $\mathrm{Li}^{1,2 \star}$, Abderr Elmoataz ${ }^{3}$, Jalal Fadili ${ }^{1}$, and $\mathrm{Su}_{\text {Ruan }}{ }^{4}$ \\ 1 GREYC-ENSICAEN, CNRS UMR 6072, 6 Bd. Maréchal Juin, 14050 Caen, France \\ 2 Dept. of Elec. \& Infor. Eng., Huazhong Univ. of Sci. \& Tech., Wuhan, P.R.China \\ 3 LUSAC, Site Universitaire, BP78, 50130 Cherbourg-Octeville, France \\ 4 Equipe Image, L.A.M.(EA2075), IUT de Troyes, 10026 Troyes, France \\ \{hua.li, abderr.elmoataz, j.fadili, su.ruan\}@greyc.ismra.fr
}

\begin{abstract}
This paper presents a curve evolution model for 3D slice-byslice image segmentation and its application in medical imaging. It is an iterative process based on the dual front evolution and the morphological dilatation to iteratively deform the initial contour towards the segmentation result. The dual front evolution model is proposed to form the new boundary by the contact position of two (or more) curves evolving in opposite directions. The fast sweeping evolution scheme is introduced for the contour evolution and the velocities for the propagation of the different curves are defined in accordance with the region-based characteristics. This model can achieve the global energy minimum and solves the disadvantages of classical level set evolution methods. Experimental results are given to illustrate the robustness of the method and its performance in precise region boundary localization and medical imaging.
\end{abstract}

\section{Introduction}

In computer vision literatures, various methods dealing with object segmentation and feature extraction are discussed [1]. Among them, active contour models [2] have emerged as a powerful tool for semi-automatic object segmentation. In recent years, many approaches have been proposed to improve the robustness and stability of active contour models [3].

Based on the Mumford-Shah minimal partition functional 4], Chan and Vese [5] proposed a new active contour model without a stopping edge-function to detect objects whose boundary are not necessarily defined by a gradient. The authors formulated this functional in terms of the level set formalism. Later, they generalized this process to treat multiple regions, and applied it to medical imaging 6]. The similar works were also proposed by Yezzi and Tsai 78 . Furthermore, under suitable assumptions, Chan and Vese's model [5] simply reduces to the $k$-means algorithm with a nonlinear diffusion preprocessing step. Then, Gibou and Fedkiw developed a hybrid numerical technique 9 that draws on

\footnotetext{
* This research work is financial supported by GRAVIR (Groupe Régional d'Action pour la Valorisation Industrielle de la Recherche) of Basse-Normandie, France.
} 
the speed and simplicity of $k$-means procedures and the robustness of level set algorithms. In [10], Xu proposed a graph cuts-based active contours approach, which combines active contour model and the optimization tool of graph cuts, for object segmentation. In his method, the graph-cuts optimization is used to iteratively deform the contour to achieve the segmentation result.

All the above interesting active contour models are implemented based on level set method [11. However, the level set method has the disadvantage of a heavy computation requirement even using the narrow band evolution. The fast marching method [12] is extremely faster than level set evolution. But within this method, the front only can move strictly positive or negative, it often exceeds the true boundary.

Recently, some improved fast marching methods were proposed for image segmentation. Cohen [13] proposed a global minimal path approach, based on fast marching method, for their active contour models. It is a global energy minimization method with complexity $O(N \log N)$, where $N$ is the number of grids. Another interesting region-growing approach was the multi-label fast marching evolution proposed by Sifakis 14 for motion analysis in video processing. An automatic stopping criterion was guaranteed due to the multiple contours marching towards the boundaries from opposite sides. Deschamps [15] also proposed an improved fast marching method in his dissertation. In these methods, the speed functions are derived from the local image information (not just gradients). The use of region's statistical information to differentiate the different front speeds has a bigger potential than the traditional speed function, which is only decided by the edge function.

In this paper, a dual front evolution model is proposed to iteratively drive the initial contour towards the segmentation result, and the fast sweeping evolution scheme is introduced in its evolution. Furthermore, the velocities for the propagation of the different contours are defined according to the region-based characteristics. A $2 \mathrm{D}$ slice-by-slice process for segmenting $3 \mathrm{D}$ image is also introduced. Our approach is simple and fast with complexity $O(N)$, in which $N$ is the number of grid points. It can easily extract the close and smooth boundary of the desired object. It is efficient and reliable, and requires very limited user intervention. Experimental results are given to illustrate the robustness of the method against noise and its performance in precise region boundary localization and medical imaging.

\section{Description of Dual Front Evolution Model}

Within the minimal path theory proposed by Cohen and coauthors [13], the surface of minimal action $U_{0}(p)$ is defined as the minimal energy integrated along a path between a starting point $p_{0}$ and any point $p$, and is shown in the following Equation (1):

$$
U_{0}(p)=\inf _{A_{p_{0}, p}}\left\{\int_{\Omega} \widetilde{P}(L(s)) d s\right\}=\inf _{A_{p_{0}, p}}\{E(L)\}
$$


Where $A_{p_{0}, p}$ is the set of all paths between $p_{0}$ and $p . L(s)$ represents a curve on a $2 \mathrm{D}$ image. $\Omega$ is its domain of definition. $E(L)$ represents the energy along the curve $L$, and $\widetilde{P}$ is the integral potential. Given the minimal action surface $U_{0}$ to $p_{0}$ and $U_{1}$ to $p_{1}$, the minimal geodesic between $p_{0}$ and $p_{1}$ is exactly the set of points $p_{g}$ that satisfy

$$
U_{0}\left(p_{g}\right)+U_{1}\left(p_{g}\right)=\inf _{p}\left\{U_{0}(p)+U_{1}(p)\right\}
$$

The minimal path between $p_{0}$ and any point $p$ in the image can be easily deduced from the action map $U$ by solving the following Eikonal Equation (3):

$$
|\nabla U|=\widetilde{P} \quad \text { with } \quad U\left(p_{0}\right)=0
$$

Now, considering all the points satisfying $U_{0}(p)=U_{1}(p)$ and the above Equation (2), at these points, the front starting from $p_{0}$ to compute $U_{0}$ first meets the front starting from $p_{1}$ to compute $U_{1}$ and the propagation stops. These points are the global minimum energy points between point $p_{0}$ and $p_{1}$. Without loss of generality, Let $X$ be a set of continuous points in the image, $U_{X}$ is the minimal action with potential $\widetilde{P}$ and starting points $\{p, p \in X\}$. Clearly, $U_{X}=\min _{p \in X} U_{p}$. Considering all the points satisfying $U_{X_{i}}(p)=U_{X_{j}}(p)$ and $U_{X_{i}}\left(p_{g}\right)+U_{X_{j}}\left(p_{g}\right)=\inf _{p}\left\{U_{X_{i}}(p)+U_{X_{j}}(p)\right\}$, these points are the global minimum energy points in the region enclosed by $X_{i}$ and $X_{j}$.

Therefore, we proposed the dual front evolution model to extract the region's boundary by finding all the points where different minimal actions $U$ are equal to any others. The besic concept of this model was introduced in [16]. In this paper, a futher detailed algorithm description is shown in Appendix. In this algorithm, the size of the narrow band can be specified by the user for a given segmentation, or a class of images. We use the morphological dilatation operator to obtain the narrow band because the iteration step size can be controlled easily by adjusting the size of the structure element and the dilatation times.

The front evolution scheme in our dual front evolution method is an extension of fast sweeping method because of its low complexity. The fast sweeping method [17] was presented by Zhao for computing the numerical solution of Eikonal equations on a rectangular grid. and gives the same result as the fast marching method but with lower complexity $O(N)$. Since the low computational cost of the fast sweeping method is maintained, the complexity of our dual front evolution method is still $O(N)$, where $N$ is the number of grid points.

\section{3D Image Segmentation Approach}

For segmenting the $3 \mathrm{D}$ image, we proposed a $2 \mathrm{D}$ slice-by-slice process [18. In this paper, we tested more synthetic images and 3D medical images to prove the validity of this $3 \mathrm{D}$ algorithm. The segmentation process includes two steps: the boundary mapping between the connective slices and the 2D boundary tracking. The flowchart in Figure 1 shows the sequence of all steps we undertake to obtain the segmentation result of $3 \mathrm{D}$ medical image. 


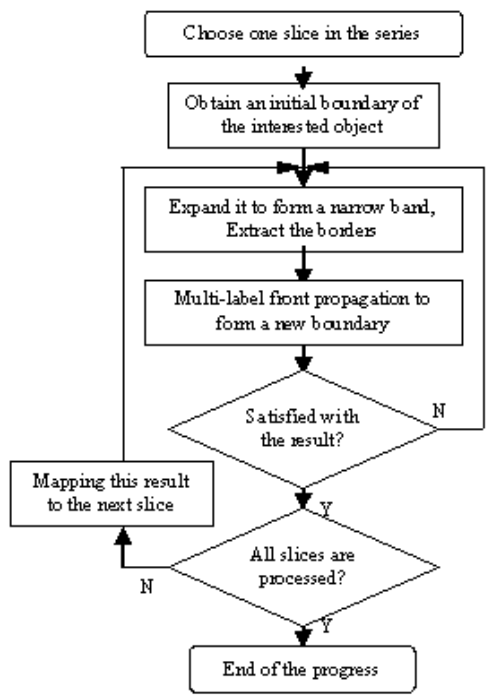

Fig. 1. Flowchart depicting the sequence of steps to segment 3D image

In medical image series, the statistics information of the corresponding regions generally change very slowly from one slice to the next, which means that the segmented region's statistics information in one slice is a good estimate of the corresponding region in the next consecutive un-segmented slice. This information is very helpful to guide the boundary tracking process. Here, we calculate the mean values $u_{i n}, u_{\text {out }}$ and the variances $\sigma_{i n}, \sigma_{\text {out }}$ of the regions inside and outside the segmented boundary in the previous slice. In the boundary tracking process of the current slice, let $l_{\text {in }}$ and $l_{\text {out }}$ be the labels of the inner and outer borders of the dilated narrow band from the mapped boundary. The propagation speeds for the labeled points $(x, y)$ are decided by the following Equations:

$$
\left\{\begin{array}{cc}
F_{\text {in }}(x, y)=\exp \left(\frac{\left|\bar{I}(x, y)-u_{\text {in }}\right|^{2}}{2 \sigma_{\text {in }}^{2}}\right)+f(\nabla I(x, y)) & \text { if } L(x, y)=l_{\text {in }} \\
F_{\text {out }}(x, y)=\exp \left(\frac{\left|\bar{I}(x, y)-u_{\text {out }}\right|^{2}}{2 \sigma_{\text {out }}^{2}}\right)+f(\nabla I(x, y)) & \text { if } L(x, y)=l_{\text {out }} \\
f(\nabla I(x, y))=\frac{1}{1+\alpha|\nabla I(x, y)|} &
\end{array}\right.
$$

where $\bar{I}(x, y)$ is the average value of the image intensity in a window of size $3 \times 3$ centered at the examined point. $|\nabla I(x, y)|$ is the image local gradient, and $\alpha$ is a constant.

In this 2D slice-by-slice segmentation process, the mapped boundary from the previous slice provides a good initialization of the boundary tracking process in current slice. In the boundary tracking process, the result of every dual front evolution step provides the initialization for the next dual front evolution. The speed function decided by the region's statistical information together with the 

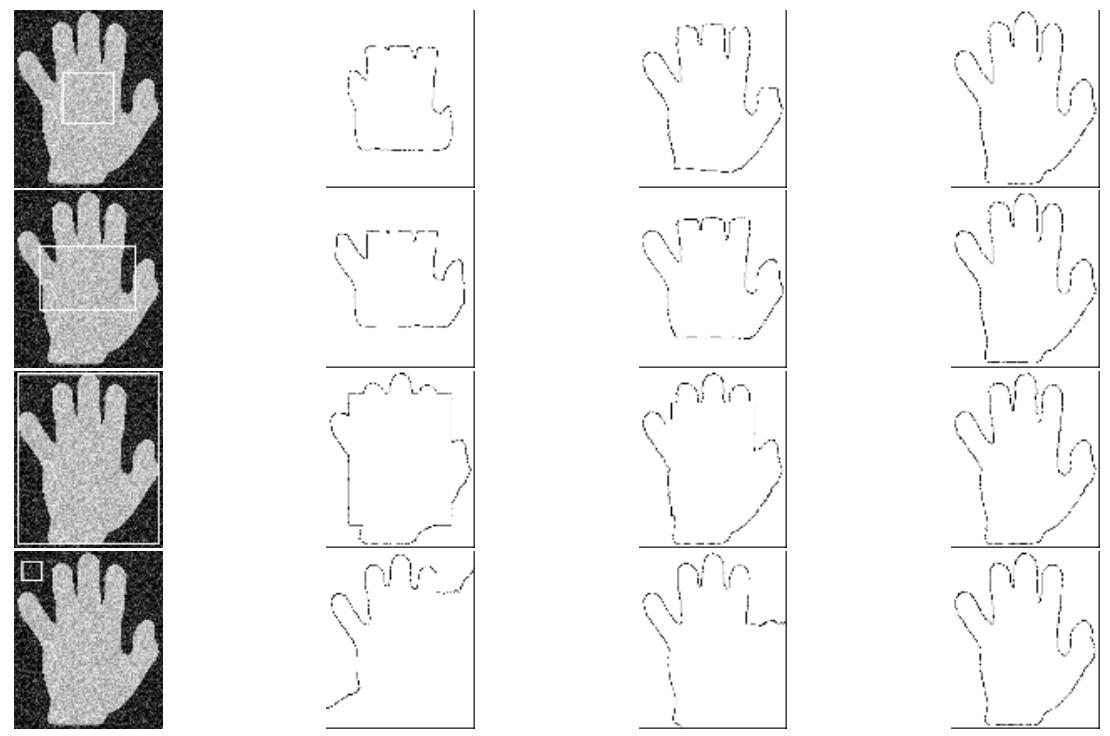

Fig. 2. The segmentation result on a hand image with different initialization

gradient information ensures an appreciate evolution. The dual front evolution method ensures an automatic evolution stopping criterion for every front propagation. The boundary tracking process stops automatically when the change between the current formed boundary and that of the previous iteration is lower than a pre-specified threshold. The total complexity of our 3D image segmentation approach is $O(M \times N)$, in which $M$ is the number of $2 \mathrm{D}$ slices and $N$ is the number of pixels in one slice.

\section{Experimental Results}

One very attractive feature associated with our method is that it automatically proceeds in the correct direction without relying upon additional inflationary terms commonly employed by many active contour algorithms. We illustrate this in Figure 2 with a noisy synthetic image of a hand. An initial contour completely contained within the interested object will flow outward towards the boundary (shown in the first row). An initial contour partially inside and partially outside the interested object will flow in both directions towards the boundary (shown in the second row). An initial contour encircling the interested object will flow inward towards the boundary (shown in the third row). And finally, an initial contour situated outside the interested object will flow outward towards and wrap around the boundary (shown in the last row). In Figure 2, the first column shows the initializing contour with the original image. The second and the third column show two intermediate steps of the algorithm. The last column shows the final segmentation curve. 

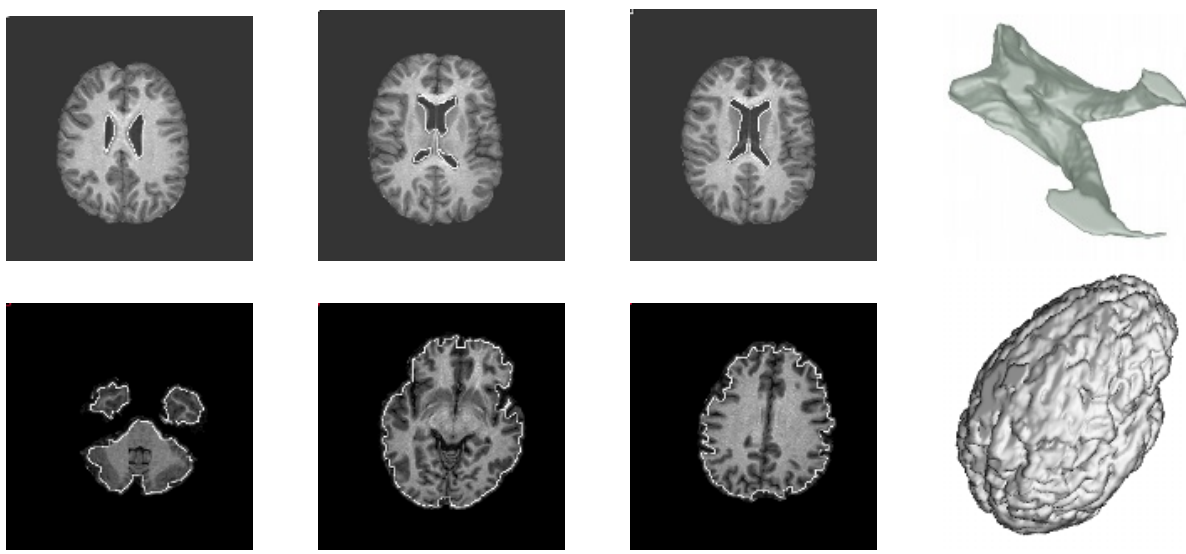

Fig. 3. The segmentation result on 3D MRI brain data

In [7], the authors also test their method on a similar hand image, but when the initial contour was outside the object, in addition to the curves that outline the boundary of the hand, there exist extraneous curves around the four corners of the image which do not correspond to image edges. This is due to the fact that their algorithm was trapped in a local minimum - a common problem faced by all algorithms which rely on gradient descent methods for minimization. However, in our experimental results on the similar image with similar initial contour, there are no extraneous curves around the corners of the image which do not correspond to image edges.

We tested our approach on two 3D medical images based on the approach described in Section 3. In the first row of Figure 3, the first three panels are the segmentation results on three different slices. The last panel is the 3D surface modeling of the segmented ventricle. In the second row of Figure 3, the first three panels are the segmentation results on three different slices. The last panel is the $3 \mathrm{D}$ surface modeling of the segmented brain. The segmentation results show the validaty of our method.

\section{Conclusions}

In this paper, a novel level set-based framework for image segmentation is presented. The dual front evolution model has been introduced to iteratively deform the initial contour towards the segmentation result and the fast sweeping scheme is introduced for the contour evolution. Our approach can detect contours with or without gradients, and provide a more global result by avoiding the disadvantage of local minima of many active contour models. Several segmentation results illustrate that this new framework is a fast, precise technique for un-supervised segmentation or labeling.

In this paper, we have demonstrated the feasibility of incorporating region information into the evolution equations for the dual front evolution model. More- 
over, future extensions of our work can be focused on the combined use of several pattern features, such as texture, gradient vector value, and color information. This can be performed using the same general framework by incorporating these distinct features into the velocity field definition. However, much more work is needed in order to test on large data sets and to improve the current algorithm.

Finally, we would like to mention that this segmentation framework using the dual front evolution model has potential applications in other image analysis domains. Examples include object tracking problems in video sequences and so on.

\section{References}

1. Munoz, X., et al.: Strategies for image segmentation combining region and boundary informa-tion. Pattern Recognition Letters 24 (2003) 375-392

2. Kass, M., Witkin, A., Terzopoulos, D.: Snakes: Active contour models. International Journal of Computer Vision 1 (1988) 321-332

3. Suri, J., Liu, K., et al.: Shape recovery algorithms using level sets in 2D/3D medical imagery: A state of the art review. IEEE Trans. on Infor. Tech. in Biomedicine 6 (2002) 8-28

4. Mumford, D., Shah, J.: Optimal approximation by piecewise smooth functions and associated variational problems. Commun. Pure Appl. Math. 42 (1989) 577-685

5. Chan, T., Vese, L.: Active contours without edges. IEEE Trans. on Image Processing 10 (2001) 266-277

6. Chan, T., Vese, L.: A multiphase level set framework for image segmentation using the mumford and shah model. International Journal of Computer Vision 50 (2002) 271-293

7. Tsai, A., Yezzi, A., Willsky, A.: Curve evolution implementation of the mumfordshah functional for image segmentation, denoising, interpolation, and magnification. IEEE Trans. on Image Processing 10 (2001) 1169-1186

8. Yezzi, A., Tsai, A., Willsky, A.: A fully global approach to image segmentation via coupled curve evolution equations. Journal of Visual Communication and Image Representation 13 (2002) 195-216

9. Gibou, F., Fedkiw, R.: A fast hybrid k-means level set algorithm for segmentation. International Journal of Computer Vision (2003)

10. Xu, N., Bansal, R., Ahuja, N.: Object segmentation using graph cuts based active contours. In: Proceedings of IEEE International Conference on Computer Vision and Pattern Recognition(CVPR). Volume 2. (2003) 46-53

11. Osher, S., Sethian, J.: Fronts propagating with curvature dependent speed: algorithms based on the Hamilton-Jacobi formulation. Journal of Computational Physics 79 (1988) 12-49

12. Sethian, J.: Fast marching methods. SIAM Review 41 (1999)

13. Cohen, L., Kimmel, R.: Global minimum for active contour models: A minimal path ap-proach. In: IEEE International Conference on CVPR (CVPR'96). (1996)

14. Sifakis, E., Tziritas, G.: Moving object localization using a multi-label fast marching algorithm. Signal Processing: Image Communication 16 (2001) 963-976

15. Deschamps, T.: Curve and Shape Extraction with Minimal Path and Level-Sets Techniques:Applications to 3D Medical Imaging. PhD thesis, UNIVERSITE de PARIS-DAUPHINE (2001) 
16. Li, H., Elmoataz, A., Fadili, J., Ruan, S.: A multi-label front propagation approach for object segmentation. In: International Conference of Pattern Recognition (ICPR2004), Combridge, UK (2004)

17. Zhao, H.: Fast sweeping method for Eikonal equation I: Distance function. http://www.math.uci.edu/ zhao (2002)

18. Li, H., Elmoataz, A., Fadili, J., Ruan, S.: 3D medical image segmentation approach based on multi-label front propagation. In: International Conference of Image Processing(ICIP2004), Singapore (2004)

\section{Appendix:}

\section{The Description of the Dual Front Evolution Scheme}

\section{Initialization:}

Label map $L$ : The initial separating contours are $B_{1}, \ldots, B_{k}$, labeling $B_{i}(1 \leq$ $i \leq k)$ as a label $l_{i}$, other rest points are labeled as -1 .

Action map $U$ : For any point $p$ of the initial contours, set $U(p)=0$; for other

\section{Input:} points, set $U(p)=\infty$.

Original image $A$ need to be segmented, the size of $A$ is $I \times J$

Initial Label map $L$

Initial Action map $U$

\section{Marching Forward Loop:}

For each point $x(i, j)$ in image $A$, calculating its new label and new action value by the ordering $i=1 \rightarrow I, j=1 \rightarrow J$ as following:

- The new label of $x$ is the label of the point having the smallest $U$ value among point $x$ and its 4-connexity neighbors.

$$
x_{\text {min }}=\left\{x \mid u(x)=\min \left(u_{i, j}, u_{i-1, j}, u_{i+1, j}, u_{i, j-1}, u_{i, j+1}\right\} \quad l_{i, j}^{\text {new }}=l\left(x_{\text {min }}\right)\right.
$$

- The new speed of point $x$ for Eikonal equation $|\nabla u(x)|=P(l(x))$ is:

$$
h_{i, j}^{n e w}=P\left(l_{i, j}^{n e w}\right)
$$

- Finding the two minimum $U$ in the 4-connexity neighbors of point $x$ :

$$
a=u_{x_{\min }}=\min \left(u_{i-1, j}, u_{i+1, j}\right) \quad b=u_{y_{\min }}=\min \left(u_{i, j-1}, u_{i, j+1}\right)
$$

- Calculating the new $U$ from the current value of its 4-connexity neighbors:

$$
\bar{u}_{i, j}= \begin{cases}\min (a, b)+h_{i, j}^{n e w} & \text { if }|a-b| \geq h_{i, j}^{n e w} \\ a+b+\frac{\sqrt{2\left(h_{i, j}^{n e w}\right)^{2}-(a-b)^{2}}}{2} & \text { if }|a-b| \leq h_{i, j}^{n e w}\end{cases}
$$

- Updating $u_{i, j}$ to be the smaller one of $\bar{u}_{i, j}$ and its current value:

$$
u_{i, j}^{\text {new }}=\min \left(u_{i, j}, \bar{u}\right)
$$

Repeat the above computation for calculating the new label and new distance value of all the points in image $A$ by the alternating ordering according the following alternating order $i=I \rightarrow 1, j=1 \rightarrow J ; i=I \rightarrow 1, j=J \rightarrow 1$; $i=1 \rightarrow I, j=J \rightarrow 1 ; i=1 \rightarrow I, j=1 \rightarrow J$.

\section{Output:}

The label map $L$ represents the final segmentation into $k$ regions $R_{k}$. 